\title{
Unleashing the mysterious link between COVID-19 and a famous childhood vasculitis: Kawasaki disease
}

\author{
Antoine Fakhry AbdelMassih ${ }^{1 *} \mathbb{D}$, Aisha Said AbdelAzeam², Aya Ayad², Aya Yasser Kamel², Ayah Khalil², \\ Basma Kotb², Dina Waheed², Esraa Menshawey², Fady Sefein², Farah Taha ${ }^{3}$, Habiba-Allah Ismail², Ibrahim Osman², \\ John Iskander ${ }^{2}$, Lama El Wakil2 ${ }^{2}$, Lara Rashad², Mariem Badr Arsanyous², Meryam El Shershaby², Mina Mansour ${ }^{2}$, \\ Mirette Ashraf ${ }^{2}$, Nada Hafez², Nadeen Mohamed Abuzeid ${ }^{2}$, Noheir Mahmoud-Nashaat AbdEISalam², \\ Nouran Gamal Hafez ${ }^{2}$, Nourhan Youssef ${ }^{2}$, Rafeef Hozaien², Rana Saeed ${ }^{2}$, Dina Kamel ${ }^{4}$, \\ Manal Ahmed AbdelHameed ${ }^{5}$ and Salma Ali $^{3}$
}

\begin{abstract}
Background: Coronavirus disease 2019 (COVID-19) emerged as a small outbreak in Wuhan rapidly progressing into the deadliest pandemic since the Spanish flu of 1918. The disease was deemed trivial in children, until the reporting, few days ago, of an emerging pediatric multi-inflammatory syndrome mimicking Kawasaki disease (KD).

Main body: This report reveals that coronaviridae were implicated in induction of several post-infectious vasculitides, namely, KD, AHEI, and HSP. This occurs in genetically susceptible individuals to vascular inflammation. Shared genetic susceptibilities between KD and CoV include genes encoding for CD 40, HLAB-15:03, and ACE. This leads to augmented inflammation with hypersecretion of cytokines especially IL-6.

Conclusion: The revealed relationships between KD and CoV can help to predict the risk of KD in COVID-19 patients through screening levels of upregulated cytokines. It might also signify that classic treatment of KD with IVIG might need to be replaced with anti-cytokine therapy in COVID-19 patients.
\end{abstract}

Keywords: COVID-19, Kawasaki disease, Shared genetic and inflammatory pathways, Upregulated inflammation

\section{Background}

Coronavirus disease 2019 (COVID-19) is caused by the seventh member of the Coronaviridea ( $\mathrm{CoV})$ family, severe acute respiratory syndrome coronavirus-2 (SARS$\mathrm{CoV}-2)$. It was declared a public health emergency of international concern on January 30, 2020, and was identified as an outbreak in March 2020. Millions of cases have been reported worldwide, with no fully effective vaccine or treatment up to this moment. It has not

\footnotetext{
* Correspondence: antoine.abdelmassih@kasralainy.edu.eg

'Pediatrics' Department, Pediatric Cardiology Unit, Cairo University Children Hospital, Faculty of Medicine, Cairo University, Kasr Al Ainy street, Cairo 12411, Egypt

Full list of author information is available at the end of the article
}

been widely identified in pediatrics, and the aggregated data on children with COVID-19 are rare. Ludvigsson et al. [1] in a systematic review stated that about $2 \%$ of the 44,672 confirmed cases of COVID-19 were children aged $0-19$ years, of which $0.9 \%$ were under 10 years of age at diagnosis. They also stated that children often have a milder form of the disease than adults with better prognosis and fewer death rates. However, New York health officials began issuing alerts on May 4, describing cases of young patients, between the ages of 2 and 15, who presented with inflammation in multiple organ systems, and features of Kawasaki disease (KD), a childhood illness of unclear origin. On May 6, the number of suspected cases increased to 64, and within that week, three 
children had died. Concurrently, France's health minister Olivier Véran told parliament that there had been some 15 cases of KD in France, although not all of the cases tested positive for COVID-19. National Health Service (NHS) of England recently warned of a similar syndrome being increasingly common in their hospital admissions $[2,3]$. To date, only one case report has been published displaying features of $\mathrm{KD}$ and testing positive for COVID-19. The 6-month-old patient case reported by Jones et al. [4] presented with fever, refusal to eat, and minimal or no respiratory symptoms as cough, rhinorrhea, and congestion. The subsequent day, she developed an erythematous not pruritic, blotchy rash, and 2 days later, her findings escalated to tachycardia, tachypnea, a fever of $38.3{ }^{\circ} \mathrm{C}$, limb-sparing conjunctivitis, and dry cracked lips. On the fifth day of her fever, she was diagnosed with KD and treated accordingly with intravenous immunoglobulins (IVIG) and high dose aspirin. Prior to admission, she tested positive for COVID-19 and was discharged 2 days later being advised to quarantine at home for 14 days while taking low-dose aspirin provided that her echocardiogram revealed no abnormalities.

It is poorly understood if both diseases occurred concurrently, or if there is rather a causal relationship between both illnesses. KD is an acute febrile illness of unknown cause that primarily affects children, especially boys younger than 5 years of age. It is one of the known causes of fever of unknown origin (FUO) in children. The disease was first described in Japan by Tomisaku Kawasaki in 1967. Typical diagnostic criteria include fever, rash, swelling of the hands and feet, peeling of skin, non-purulent conjunctival injection, lymphadenopathy, and coronary artery abnormalities $[4,5]$.

In this review, we are analyzing previously available data to demonstrate the possible relationship between KD and COVID-19. We hypothesize that such a link might be post-infectious in genetically susceptible patients. We also hypothesize that this relationship operates through an increased tendency for vascular inflammation and upregulated cytokine profile. Finally, yet importantly, we are discussing the possible need for treatment modification of $\mathrm{KD}$ developing in the midst of COVID-19.

\section{Main text}

$\mathrm{KD}$, a possible post-infectious illness, is CoV implicated? Evidence of several respiratory infections inducing $K D$

According to various studies, the most accepted theory for the etiology of KD is the exposure to one or more infectious agent(s) will trigger an inflammatory response in genetically predisposed individuals [6, 7]. In 2000, Rowley et al. [8] discovered that during acute $\mathrm{KD}$, immunoglobulin A (IgA) plasma cells infiltrate vascular tissues (as coronary walls) and non-vascular tissues, primarily the trachea which highly suggest the route of entry of the KD etiologic agents is respiratory. Also, in another study, Rowley et al. reported oligoclonal IgA antibodies in bronchial epithelium from patients with acute KD in addition to the macrophages found in inflamed coronary artery tissues. This highly supports the idea that the immune response triggered by $K D$ is antigen-driven (hence oligoclonal) [9]. Intracytoplasmic inclusion bodies were also detected indicating a viral etiology for KD [10]. Further investigation of the infectious etiology of KD was done by Chang et al. [11] who found an association between viral infections, such as respiratory viruses, and the development of $\mathrm{KD}$. In addition, Kim et al. [12] stated that a viral infection most likely precedes $\mathrm{KD}$, supporting the theory that $\mathrm{KD}$ has a postinfectious etiology. Many studies thereof set out to identify the causative organisms triggering $\mathrm{KD}$, such as Negro et al. [13], citing a higher incidence of developing all six major criteria of $\mathrm{KD}$ in patients who had a recent or active parvovirus B19 infection. Weng et al. [14] also noted a significant incidence of KD in children with previously documented enterovirus infection.

The theory that KD is a post-infectious illness has been confirmed by Maggio et al. [15], who found KD and Kawasaki shock syndrome in two siblings after documented parvovirus infections. Schnaar et al. [16] reported similar findings in cousins who were in close contact following parainfluenza type 3 viral infections, and Lee et al. [17] indicated the involvement of varicella-zoster virus in two sisters. Bocavirus was also implicated by Bajolle et al. [18], while the involvement of adenovirus has only been postulated but not proven by Shike et al. [19] due to similar clinical features. The possibility of KD being post-infectious is also proved by Embil et al. [20], who managed to extract adenovirus from the mesenteric lymph nodes in two KD patients post mortem.

\section{Coronaviridae epidemiology tracing in $K D$}

There have been studies suggesting the possible involvement of $\mathrm{CoV}$ in KD. Serological tests were performed for human coronavirus 229E (HCoV-229E) and supported that a particular strain of the virus might play a role in the etiology of KD [21]. Other studies focused on coronavirus OC43/HKU1 [22] and a case of COVID-19 [23]. While there was also initial speculation of the involvement of COV-NL63, these claims have since been refuted.

Evidence-based immunopathological studies done by Rowley et al. [24] suggest that KD is caused by a respiratory infectious agent. Furthermore, the suspected seasonality of KD roughly coincides with that of the most common respiratory HCoVs [25]. However, it is too 
early to tell if such seasonality also applies to COVID19. Further research is necessary, but the factors above suggest a probable connection between $\mathrm{CoV}$ and $\mathrm{KD}$.

\section{Association of coronaviridae with other types of vasculitides}

CoV has a synergistic relationship with patients manifesting post-infectious vasculitis, as vasculitis can be either a risk factor or post-infectious outcome to coronavirus. Owing to the fact that vasculitis can be a consequence to coronavirus, a case report presented by Chesser et al. [26] showed the recurrence of acute hemorrhagic edema of infancy (AHEI) in an 8-monthold female who previously tested positive for coronavirus NL63 via Nucleic Acid Amplification test (NAAT). AHEI is a rare disease affecting infants younger than 24 months, and is known as a benign type of leukocytoclastic vasculitis, and is considered a variant of Henoch Schoenlein Purpura (HSP).

Additionally, from January 2013 till December 2016, the Korean Centers for Disease Control and Prevention gathered information on pathogens causing acute respiratory and enteric infectious diseases, to study the seasonal tendencies in pediatric HSP, and its correlation with the outbreak of infectious diseases. The seasonal analysis indicated that a considerable number of HSP cases (5252, 31.0\%, and 4437) were diagnosed in spring and winter respectively, while the smaller number was in summer (3224, 19.0\%). This supports that HSP cases are mostly reported in colder months, coinciding with the winter infectious outbreaks as coronavirus [27, 28]. Correlation analysis between Henoch-Schönlein purpura occurrence and prevalence of viral infections in the former study indicated that there were 2184 positive cases of coronavirus, with a $p$ value $(0.005)$ and correlation coefficient (0.194). The etiology of HSP remains quite vague; however, upper respiratory tract infections preceded 30\% of the HSP cases reported in Spain [27].

A report from China [29] supported the vascular effect of severe acute respiratory syndrome (SARS), by investigating the histopathology from the autopsy of three patients who died from SARS. It showed systemic vasculitis with infiltration of small blood vessels by monocytes, lymphocytes, focal necrosis, and edema in multiple organs including the lung, heart, brain, liver, and kidneys.

\section{Endothelial inflammation in both disorders}

There is an increasing body of evidence that KD involves widespread endothelial dysfunction, such endothelial dysfunction might be induced by reactive oxygen species. It endothelial dysfunction and inflammation in patients with KD is not limited to coronary endothelium but involves renal and mesenteric vascular endothelium and might persist long after resolution of KD [30, 31].

Moreover, there is accumulating evidence showing that the multi-organ failure reported in COVID-19 patients is due mainly to the inflammatory response caused by viral infection of the endothelium rather than to the direct action of the virus. Postmortem examination of vascular samples of affected patients by Varga et al. revealed significant inflammation of the vascular endothelium. This was confirmed by Escher et al., who stated that upregulated cytokines in COVID-19 induce endothelial active inflammation. This might explain the micro-thrombi developing in the lungs of affected patients and the subsequent need of anti-platelets and anticoagulant therapy in most critical patients [32].

\section{Similar geographic distributions with shared genetics of upregulated inflammation}

It is known that KD has higher rates in the far east, namely, in Japan, Korea, Taiwan, and intermediate rates in China, the Philippines, and other Asian countries [33]. Coronavirus outbreaks were first recognized in the Far East, which from there they spread to the whole world. As SARS-CoV started in China, HKU1-CoV started in Hong Kong and Middle East Respiratory Syndrome (MERS)-CoV in the Middle East [34]. Lastly, COVID-19 started in Wuhan, China [35]. The similar geographic and racial distributions of both disorders raise the suspicion of shared genetic susceptibilities in the affected populations.

\section{ACE I/D polymorphism}

Pooled analysis suggests that the angiotensin converting enzyme insertion/deletion (ACE I/D) polymorphism was significantly associated with KD risk specifically in subgroup analysis by sample size $>200$ as demonstrated by Pan et al. [36].

Delanghe et al. mentioned that ACE1 I/D polymorphism may be regarded as a confounder in the spread of COVID-19, and the outcome of the infection in various European populations, where the log-transformed prevalence of COVID-19 infections inversely correlates with the ACE I/D allele frequency. It is worth mentioning that China and Korea, which were initially severely hit by the virus, are characterized by low D allele frequencies [37].

\section{Major histocompatibility complex and human leucocyte antigen polymorphism}

Major histocompatibility complex (MHC) class I genes and human leukocyte antigen (HLA) A, B, and C individual genetic variation may affect the severity and susceptibility to SARS-CoV-2 as well as KD.

A comprehensive in silico analysis of viral peptideMHC class I binding affinity across 145 HLA genotypes 
for all SARS-CoV-2 peptides was conducted by Nguyen et al. showed that HLA-B15:03 has the greatest capacity to present highly conserved SARS-CoV-2 peptides that are shared among common human coronaviruses. This suggests that HLA-B15:03 could strongly activate T-cell mediated immunity, with subsequent induction of vascular inflammation [38].

As for HLA variability affecting KD, Oh et al. conducted an analysis of the polymorphisms of HLA types. It proved that there was a significant increase in the frequency of HLA-B15:03, along with HLA-B35, HLAB75, and HLA-Cw09 alleles in patients with KD compared with the control healthy group. When the patients with KD were divided into two subgroups, with or without $C C$, the KD patients without $C C$ showed a significantly increased frequency of HLA-B35, HLA-B75, and HLA-Cw09 alleles as opposed to a decrease in HLA-A26 when compared with the healthy control group. HLAB15, the shared HLA between SARS and KD, was linked to a higher risk of endothelial inflammation and coronary aneurysms [39].

\section{Ligand gene of cluster of differentiation 40}

Cluster of differentiation $40(\mathrm{CD} 40)$ is a co-stimulatory protein found on antigen-presenting cells and is required for their activation. The binding of cytotoxic $\mathrm{T}$ cells on $\mathrm{TH}$ cells to CD40 activates antigen-presenting cells and induces a variety of downstream effects. Such effects have been linked to increased risk of vasculitis and endothelial dysfunction.

Kumrah et al. stated that CD40LG (ligand gene of CD 40) has been found as an independent predictor of $\mathrm{KD}$ occurrence and KD progression [40].

SARS-CoV-2 interaction with human cells has been thought to occur exclusively through angiotensin converting enzyme 2 (ACE2). Recently, Dakal et al. stated that SARS-CoV-2 attachment to host cells is possibly mediated also by a sequence of tripeptide consisting of arginine, glycine, and aspartate (RGD). Integrin binding would activate the expression of CD40LG; CD40LG augmented expression increases the likelihood of inflammatory sequelae of COVID-19 [41].

\section{Cytokine profile in both disorders \\ Innate immunity cytokines: IL2, IL10, and TNF}

Interlukin-2 (IL-2), interlukin-10 (IL-10), and tumor necrosis factor-alpha (TNF- $\alpha$ ) were measured in a study involving intensive care unit (ICU) patients with COVID-19 showed higher significant levels than those who were not admitted [42]. A previously published paper proved that IL-2 receptor level was higher in KD patients than in control group [43].

Moreover, Hsueh et al. proved that IL-10 promotor polymorphism at position 592 (IL-10-592*A allele) located on chromosome 1q31-q32 with KD genetic susceptibility in Taiwanese patients [44]. Also, according to a study on KD pathogenesis, children suffering from the disease presented with significantly elevated levels of TNF $\alpha$. In addition, anti-TNF $\alpha$ was tested in the treatment of KD and showed significant improvement [45].

\section{TGF- $\beta$}

Simultaneously, the role of transforming growth factor beta (TGF- $\beta$ ) in COVID-19 promotes viral entry into the cells [46]. In the context of Kawasaki, TGF- $\beta$ affects the genetic susceptibility of $\mathrm{KD}$, regarding the outcome and likelihood for persistent coronary aneurysms. Furthermore, TGF- $\beta$ modulates the balance of pro-inflammatory/ anti-inflammatory $\mathrm{T}$-cells through the expression of the Forkhead box P3 (FOXP3), which affects the differentiation function and survival of $\mathrm{CD} 4+\mathrm{CD} 25+$ regulatory $\mathrm{T}$ cells. Additionally, it causes coronary artery aneurysm by promoting the production of myofibroblast [44].

\section{IL-6 a common effector between KD and COVID-19}

Initiated by tumor necrotic factor alpha (TNF- $\alpha$ ), IL-6, a pro-inflammatory cytokine, and an anti-inflammatory myokine are immediately stimulated and secreted in a state of infection or tissue injury, which greatly contributes to the hosts' defense mechanisms [47, 48]. This is via activation of the IL- 6 gene, which will result in the arousal of acute-phase responses and immune reactions as proven by experiments done in vivo and vitro [47].

The contribution of IL-6 with regards to KD has not been clearly understood. It is presumed that IL- 6 directly promotes maturation of megakaryocytes, thus responsible for the thrombocytosis witnessed in the disease. Elevated IL-6 inhibits T-helper 1 (Th1) cell differentiation and promotes T-helper 2 (Th2) cell activation, which increases Th2 cytokines; as a result, polyclonal B cell producing autoantibodies will be stimulated. Not only does it contribute to the acute inflammatory reaction of $\mathrm{KD}$, but it also plays a role in its vasculitis component by activation of antibody-mediated endothelial damage $[49,50]$.

According to several studies, there are increased levels of serum IL-6 in children with KD, especially during the acute phase in comparison to the subacute phase. KD patients with higher levels of IL- 6 are more prone to developing coronary artery lesions (CALs), which goes in accordance with Alolika et al. who states that during pre- and post-IVIG treatment, IL-6 levels were drastically greater in KD patients with CALs $(143.60 \pm 99.72$ $\mathrm{pg} / \mathrm{dL})$ than those without $(52.90 \pm 36.46 \mathrm{pg} / \mathrm{dL})$. IL-6 levels were also predictive of the occurrence of coronary aneurisms, with a sensitivity of $83.3 \%$ and a specificity of 95.8\% [51].

$\mathrm{Wu}$ and colleagues mention a unique correlation between increased IL-6 levels and IVIG resistance, 
suggesting that IL-6 could be a reliable prognostic marker for IVIG resistance. There is a clear difference in the levels of IL- 6 in patients without IVIG resistance $(64.1 \pm 51.5 \mathrm{pg} / \mathrm{dL})$ than those with resistance $(184 \pm$ $65.0 \mathrm{pg} / \mathrm{dL}$ ) [52]. In 2017, a study revealed that elevated IL-6 is an independent risk factor of IVIG nonresponsiveness with a sensitivity of $76.19 \%$ and a specificity of $61.59 \%$. This is in accordance with Nandi et al., which advocate that IL- 6 levels should be used to determine the method of treatment in KD patients. The IVIG mechanism of inflammatory modulation may be largely dependent on Th2 cytokines including IL- 6 . Whether or not IL-6 is a key factor in the pathogenesis of KD is not crystal clear and requires extensive research to fully understand. As for what is understood, serum IL-6 has proved to be a novel marker for the prediction of coronary artery involvement and resistance to IVIG [53].

In COVID-19, three pathways of inflammations have been identified, namely, the ACE2 and JAK/STAT and Notch pathways. Both pathways have been linked to an increase in IL-6. Evidence suggests that IL-6 levels have been linked to most of the multi-system complications of COVID-19. An elevated response of IL-6-induced severe respiratory distress. Thus, our results suggest that serial measurement of circulating IL-6 levels may be important in identifying disease progression among
COVID-19-infected patients. AbdelMassih et al. suggested that IL- 6 might be a possible inducer of COVID19 myocardial complications. Therefore, it is reasonable to suggest that immediate initial evaluation of IL-6 level be performed upon hospital admission of COVID-19 pediatric patients, due to its potential benefits to assess worsening clinical features, and disease progression in COVID-19 [54-61].

\section{Anti-IL6 therapy and its possible role in COVID-19 induced KD}

The repurposed use of anti-IL-6 inhibitors, whether siltuximab, sarlizumab, or tocilizumab, has been identified as a potential therapy for COVID-19 critical patients. Such inhibitors have been linked to decreased oxygen needs and decreased number of mechanical ventilation days compared to symptomatic treatment. This has led to Food and Drug Administration (FDA) approval of several clinical trials involving the use of those drugs for the treatment of critically ill COVID-19 patients [62].

In KD, cytokine-blockade therapies have been suggested as superior therapies to IVIG. A clinical trial of anakinra, IL-1 $\beta$-blockade therapy, was recently launched (ClinicalTrials.gov number, NCT02390596). IL-6 plays an important role in tissue regeneration, and the targeted blockade of IL-6 could disrupt this process. In

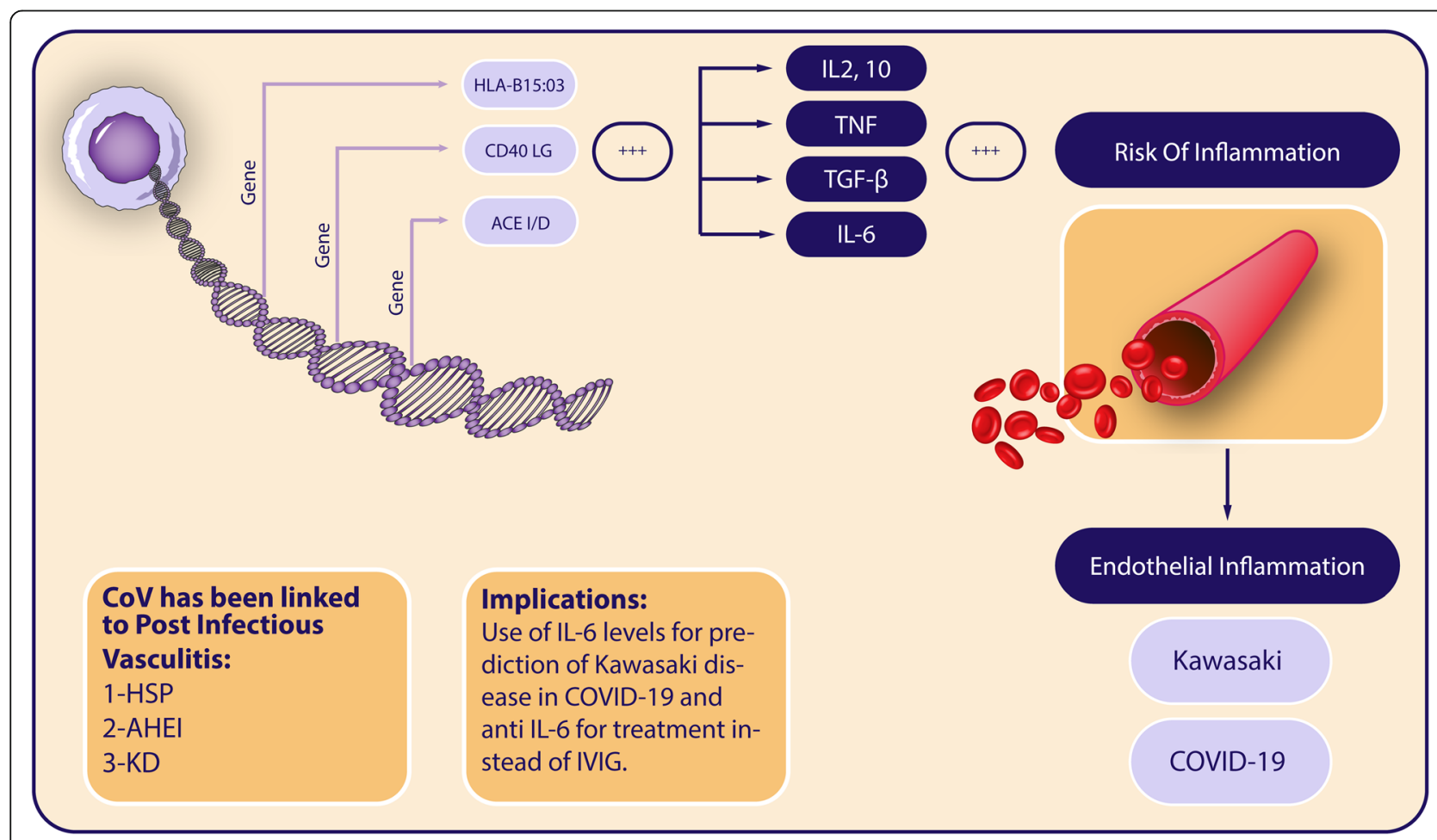

Fig. 1 Summary of possible linkage pathways between Kawasaki disease and COVID-19. HLA, human leukocyte antigen; CD4, cluster of differentiation 4; ACE, angiotensin-converting enzyme; IL, interleukin; TNF, tumor necrosis factor; TGF-beta, transforming growth factor beta; HSP, Henoch-Schonlein purpura; AHEl, acute hemorrhagic edema of infancy; KD, Kawasaki disease; IVIG, intravenous immunoglobulin 
addition, IL- 6 contributes to the reduction of neutrophil trafficking into the arterial wall. Tocilizumab can inhibit vascular endothelial growth factor, which enhances proliferation and migration of endothelial cells, and contributes to the remodeling of coronary-artery aneurysms [63]. Thus, the use of tocilizumab in the context of COVID-19 patients might be needed, in IVIG resistant cases or even prior to the administration of IVIG.

Figure 1 is a summary of the possible shared mechanisms of KD and COVID-19 and the implications of such findings.

\section{Conclusion}

This is by far, the earliest report to study the linkage between the commonest childhood vasculitis, KD, and COVID-19. There is strong past and present body of evidence that $\mathrm{CoV}$ can induce a state of post-infectious vasculitis in genetically predisposed individuals. This occurs through upregulated baseline levels of inflammation. The knowledge of that can help in tailoring the therapeutic strategies in patients presenting with KD concurrently with COVID-19. The early screening of IL-6 levels in pediatric COVID-19 patients can be of utmost importance in detection of subsequent risk of $\mathrm{KD}$. The use of IL-6 inhibitors in those developing KD can have a superior effect that the classic IVIG therapy.

Prospective studies are needed to confirm or refute such findings.

\section{Abbreviations}

Aspirin: Acetylsalicylic acid; AHEl: Acute hemorrhagic edema of infancy; ACE: Angiotensin converting enzyme; ACE I/D: Angiotensin converting enzyme insertion/deletion; CALs: Coronary artery lesions; CC: Coronary complications; CD: Cluster of differentiation; CD40LG: Ligand gene of CD 40; CoV: Coronaviridae; CRP: C-reactive protein; ESR: Erythrocyte sedimentation rate; FDA: Food and Drug Administration; FOXP3: Forkhead/winged-helix transcription factor P3; FUO: Fever of unknown origin; HCoV: Human coronaviridae; HLA: Human leucocyte antigen; HSP: Henoch-Schönlein purpura; ICU: Intensive care unit; Ig: Immunoglobulin; IL: Interleukin; IVIG: Intravenous immunoglobulins; JAK: Janus kinase; KD: Kawasaki disease; LG: Ligand; MHC: Major histocompatibility complex; NAAT: Nucleic acid amplification test; RGD: Arginine, glycine, and aspartate tripeptide; SARS: Severe acute respiratory syndrome; SARS-CoV-2: Severe acute respiratory syndrome coronaviridae-2; SNP: Single nucleotide polymorphism; STAT: Signal transducer and activator of transcription; Th1: T-helper cell 1; Th2: T-helper cell 2; TGF $\beta$ : Transforming growth factor beta; TNF-a: Tumor necrosis factor-alpha

\section{Acknowledgements}

To our families, and to every doctor who is fighting in the frontline against COVID-19. On behalf of all co-authors, I wanted to thank Prof. Hala Salah, dean of the faculty of medicine, and Prof. Neveen Soliman, the vice dean for research issues, for their tremendous support for our work. I also wanted to thank the crew of Pediatric Cardiology Unit, Cairo University, headed by Prof. Hala Agha and co-headed by Prof. Sonia ElSaeidi and Prof. Amal El-Sisi for their valuable comments during the writing of this work. We wanted as well to acknowledge the editorial board and production of Egyptian Pediatric Association Gazette headed by Prof. Khaled BahaaEIDin for fast tracking this work. A word of gratefulness should go to Pixagon Agency headed by the great artist Dr. Nadine ElHusseiny for providing us with her game-changing artwork. Finally, yet importantly, I wanted as a first author to thank all the families of the students and residents who co-authored this paper, for beautifully raising them. I end the acknowledgement with a dedication of this work to my mother, my wife, and my three children, Maria, George, and Marc; may I always be able to make them proud of me.

\section{Authors' contributions}

AFA, $\mathrm{HI}, \mathrm{RH}$, and AYK were a major contributor to the conception of the idea. All authors have contributed to each of the following: design of the structure of the review article sections, acquisition and analysis of data, and drafting of the manuscript. All authors read and approved the final manuscript.

\section{Funding \\ None}

Availability of data and materials

Not applicable

Ethics approval and consent to participate

Not applicable

Consent for publication

Not applicable

\section{Competing interests}

The authors declare that they have no competing interests.

\section{Author details}

${ }^{1}$ Pediatrics' Department, Pediatric Cardiology Unit, Cairo University Children Hospital, Faculty of Medicine, Cairo University, Kasr Al Ainy street, Cairo 12411, Egypt. 'Students' and Interns' Research Program (Research accesibility team), Faculty of Medicine, Cairo University, Al-Saray Street, El Manial, PO BOX: 11956, Cairo, Egypt. ${ }^{3}$ Resident training Program, Faculty of Medicine, Cairo University, Al-Saray Street, El Manial, PO BOX: 11956, Cairo, Egypt. ${ }^{4}$ Pediatrics' Department, Faculty of Medicine, Ain Shams University, Abbassyia, PO BOX: 11566, Cairo, Egypt. ${ }^{5}$ Pediatrics' Department, Faculty of Medicine, Cairo University, Al-Saray Street, El Manial, PO BOX: 11956, Cairo, Egypt.

Received: 14 May 2020 Accepted: 28 May 2020

Published online: 01 July 2020

\section{References}

1. Ludvigsson JF (2020) Systematic review of COVID-19 in children shows milder cases and a better prognosis than adults. Acta Paediatr Int J Paediatr: 1088-1095. https://doi.org/10.1111/apa.15270

2. WHO (2020) Coronavirus disease. World Health Organ 2019:2633

3. Laboratories C, Surveillance NS (2020) Outpatient and Emergency Department Visits Severe Disease Summary of Laboratory Testing Results Reported to CDC *

4. Jones VG et al (2020) Pre-publication Release COVID-19 and Kawasaki Disease: Novel Virus and Novel Case Pre-publication Release Pre-publication Release. Hosp Pediatr Cit. https://doi.org/10.1542/hpeds.2020-0123

5. Barron KS (2000) Kawasaki disease. In: Inflammatory Diseases of Blood Vessels. https://doi.org/10.1201/b14012

6. Burgner D, Harnden A (2005) Kawasaki disease: What is the epidemiology telling us about the etiology? Int J Infect Dis. https://doi.org/10.1016/j.jijd. 2005.03.002

7. Sharma D, Singh S (2015) Kawasaki disease - A common childhood vasculitis. Indian J Rheumatol 10:S78-S83

8. Rowley AH et al (2000) IgA Plasma Cell Infiltration of Proximal Respiratory Tract, Pancreas, Kidney, and Coronary Artery in Acute Kawasaki Disease. J Infect Dis 182:1183-1191

9. Rowley AH, Shulman ST, Spike BT, Mask CA, Baker SC (2001) Oligoclonal IgA Response in the Vascular Wall in Acute Kawasaki Disease. J Immunol 166: 1334-1343

10. Rowley AH et al (2005) Cytoplasmic Inclusion Bodies Are Detected by Synthetic Antibody in Ciliated Bronchial Epithelium during Acute Kawasaki Disease. J Infect Dis 192:1757-1766

11. Chang LY et al (2014) Viral infections associated with Kawasaki disease. J Formos Med Assoc 113:148-154 
12. Kim GB et al (2014) Evaluation of the temporal association between Kawasaki disease and viral infections in South Korea. Korean Circ J 44:250254

13. Nigro $\mathrm{G}$ et al (1994) Active or recent parvovirus B19 infection in children with Kawasaki disease. Lancet 343:1260-1261

14. Weng KP et al (2018) Enterovirus Infection and Subsequent Risk of Kawasaki Disease: A Population-based Cohort Study. Pediatr Infect Dis J 37:310-315

15. Maggio MC et al (2019) Kawasaki disease triggered by parvovirus infection: An atypical case report of two siblings. J Med Case Rep 13:104

16. Schnaar DA, Bell DM (1982) Kawasaki Syndrome in Two Cousins With Parainfluenza Virus Infection. Am J Dis Child 136:554-555

17. Lee D-H, Huang H-P (2004) Kawasaki disease associated with chickenpox: report of two sibling cases. Acta Paediatr Taiwan 45:94-96

18. Bajolle F et al (2014) Markers of a recent bocavirus infection in children with Kawasaki disease: 'A year prospective study'. Pathol Biol 62:365-368

19. Shike $\mathrm{H}$ et al (2005) Adenovirus, Adeno-associated virus and kawasaki disease. Pediatr Infect Dis J 24:1011-1014

20. Embil JA, McFarlane ES, Murphy DM, Krause WW, Stewart HB (1985) Adenovirus type 2 isolated from a patient with fatal Kawasaki disease. Can Med Assoc J 132:1400

21. Shirato $\mathrm{K}$ et al (2014) Possible involvement of infection with human coronavirus 229E, but not NL63, in Kawasaki disease. J Med Virol 86:21462153

22. Giray $T$ et al (2016) Four cases with kawasaki disease and viral infection: Aetiology or association? Infez Med 24:340-344

23. Jones VG et al (2020) COVID-19 and Kawasaki Disease: Novel Virus and Novel Case. Hosp Pediatr. https://doi.org/10.1542/hpeds.2020-0123

24. Rowley AH et al (2004) Detection of Antigen in Bronchial Epithelium and Macrophages in Acute Kawasaki Disease by Use of Synthetic Antibody. J Infect Dis 190:856-865

25. McIntosh K (2005) Coronaviruses in the Limelight. J Infect Dis 191:489-491

26. Chesser H, Chambliss JM, Zwemer E (2017) Case Report Acute Hemorrhagic Edema of Infancy after Coronavirus Infection with Recurrent Rash. https:// doi.org/10.1155/2017/5637503

27. Hwang HH, Lim IS, Choi BS, Yi DY (2018) Analysis of seasonal tendencies in pediatric Henoch-Schönlein purpura and comparison with outbreak of infectious diseases. Medicine (United States) 97

28. Gedalia A (2004) Henoch-Schönlein Purpura. Curr Rheumatol Rep 6:195-202

29. Ding $Y$ et al (2003) The clinical pathology of severe acute respiratory syndrome (SARS): a report from China. J Pathol 200:282-289

30. Dhillon $\mathrm{R}$ et al (1996) Endothelial dysfunction late after Kawasaki disease. Circulation. https://doi.org/10.1161/01.CIR.94.9.2103

31. Ishikawa T, Seki K (2018) The association between oxidative stress and endothelial dysfunction in early childhood patients with Kawasaki disease. BMC Cardiovasc Disord 18:1-8

32. Poor HD et al (2020) COVID-19 Critical IIIness Pathophysiology Driven by Diffuse Pulmonary Thrombi and Pulmonary Endothelial Dysfunction Responsive to Thrombolysis. medRxiv. https://doi.org/10.1101/2020.04.17 20057125

33. Rowley AH, Shulman ST (2018) The epidemiology and pathogenesis of Kawasaki Disease. Front Pediatr 6:1-4

34. Su S et al (2016) Epidemiology, Genetic Recombination, and Pathogenesis of Coronaviruses. Trends Microbiol 24:490-502

35. Rothan HA, Byrareddy SN (2020) The epidemiology and pathogenesis of coronavirus disease (COVID-19) outbreak. J Autoimmun. https://doi.org/10, 1016/j.jaut.2020.102433

36. Pan Y, Lu H (2017) Angiotensin-converting enzyme insertion/deletion polymorphism and susceptibility to Kawasaki disease: A meta-analysis. Afr Health Sci 17:991-999

37. Delanghe JR, Speeckaert MM, De Buyzere ML (2020) COVID-19 infections are also affected by human ACE1 D/I polymorphism. Clin Chem Lab Med:1-2. https://doi.org/10.1515/cclm-2020-0425

38. Nguyen A et al (2020) Human leukocyte antigen susceptibility map for SARS-CoV-2. J Virol. https://doi.org/10.1128/jvi.00510-20

39. Oh JH et al (2008) Polymorphisms of human leukocyte antigen genes in Korean children with Kawasaki disease. Pediatr Cardiol 29:402-408

40. Kumrah R, Vignesh P, Rawat A, Singh S (2020) Immunogenetics of Kawasaki disease. Clin Rev Allergy Immunol. https://doi.org/10.1007/s12016-02008783-9

41. Chand Dakal T et al (2020) Mechanistic basis of co-stimulatory CD40-CD40L ligation mediated regulation of immune responses in cancer and autoimmune disorders. Immunobiology. https://doi.org/10.1016/j.imbio. 2019.151899

42. Gong J et al (2020) Correlation Analysis Between Disease Severity and Inflammation-related Parameters in Patients with COVID-19 Pneumonia. medRxiv:2020.02.25.20025643. https://doi.org/10.1101/2020.02.25.20025643

43. Teraura $\mathrm{H}$ et al (2017) The serum concentration of soluble interleukin-2 receptor in patients with Kawasaki disease. Ann Clin Biochem 54:209-213

44. Del Principe D et al (2017) Pathogenetic determinants in Kawasaki disease: the haematological point of view. J Cell Mol Med 21:632-639

45. Xue LJ et al (2017) Effect and Safety of TNF Inhibitors in ImmunoglobulinResistant Kawasaki Disease: a Meta-analysis. Clin Rev Allergy Immunol. https://doi.org/10.1007/s12016-016-8581-4

46. Chen W (2020) A potential treatment of COVID-19 with TGF- $\beta$ blockade. Int J Biol Sci 16:1954-1955

47. Tanaka T, Narazaki M, Kishimoto T (2014) IL-6 in Inflammation, Immunity, and Disease. 6:1-16

48. Kuo HC et al (2010) Serum albumin level predicts initial intravenous immunoglobulin treatment failure in Kawasaki disease. Acta Paediatr Int J Paediatr. https://doi.org/10.1111/j.1651-2227.2010.01875.x

49. Ueno $Y$ et al (1989) The acute phase nature of interleukin 6: studies in Kawasaki disease and other febrile illnesses. Clin Exp Immunol 76:337-342

50. Lin CY, Lin CC, Hwang B, Chiang BN (1993) Cytokines predict coronary aneurysm formation in Kawasaki disease patients. Eur J Pediatr 152:309-312

51. Gauldie J, Richards C, Harnish D, Lansdorp P, Baumann H (1987) Interferon beta 2/B-cell stimulatory factor type 2 shares identity with monocytederived hepatocyte-stimulating factor and regulates the major acute phase protein response in liver cells. Proc Natl Acad Sci U S A 84:7251-7255

52. Wu Y et al (2019) Interleukin-6 is prone to be a candidate biomarker for predicting incomplete and IVIG nonresponsive Kawasaki disease rather than coronary artery aneurysm. Clin Exp Med. https://doi.org/10.1007/s10238-01800544-5

53. Nandi A, Pal P, Basu S (2019) A comparison of serum IL6 and CRP levels with respect to coronary changes and treatment response in Kawasaki disease patients: a prospective study. Rheumatol Int 39:1797-1801

54. Fan C, Li K, Ding Y, Lu WL, Wang J (2020) ACE2 Expression in Kidney and Testis May Cause Kidney and Testis Damage After 2019-nCoV Infection. medRxiv:2020.02.12.20022418. https://doi.org/10.1101/2020.02.12.20022418

55. Booz GW, Day JNE, Baker KM (2002) Interplay between the cardiac renin angiotensin system and JAK-STAT signaling: Role in cardiac hypertrophy, ischemia/reperfusion dysfunction, and heart failure. J Mol Cell Cardiol 34: $1443-1453$

56. Qi YF et al (2016) Angiotensin-converting enzyme 2 inhibits high-mobility group box 1 and attenuates cardiac dysfunction post-myocardial ischemia. J Mol Med. https://doi.org/10.1007/s00109-015-1356-1

57. Conti $\mathrm{P}$ et al (2020) Induction of pro-inflammatory cytokines (IL-1 and IL-6) and lung inflammation by COVID-19: anti-inflammatory strategies. J Biol Regul Homeost Agents. https://doi.org/10.23812/CONTI-E

58. Rizzo P et al (2020) COVID-19 in the heart and the lungs: could we "Notch" the inflammatory storm? Basic Res Cardiol 115:1-8

59. AbdelMassih, A. et al. Is it infection or rather vascular inflammation? Gamechanger insights and recommendations from patterns of multi-organ involvement and affected subgroups in COVID-19, Cardiovascular Endocrinology \& Metabolism: June 11, 2020 - Volume Publish Ahead of Print - Issue - https://doi.org/10.1097/XCE.0000000000000211

60. AbdelMassih, A. et al. Obese communities among the best predictors of COVID-19-related deaths. Cardiovascular Endocrinology \& Metabolism: June 11, 2020 - Volume Publish Ahead of Print - Issue - https://doi.org/10.1097/ XCE.0000000000000218

61. AbdelMassih, A. et al. Possible molecular and paracrine involvement underlying the pathogenesis of COVID-19 cardiovascular complications, Cardiovascular Endocrinology \& Metabolism: April 20, 2020 - Volume Publish Ahead of Print - Issue - https://doi.org/10.1097/XCE.0000000000000207

62. Luo $P$ et al (2020) Tocilizumab treatment in COVID-19: A single center experience. J Med Virol. https://doi.org/10.1002/jmv.25801

63. Nozawa T, Imagawa T, Ito S (2017) Coronary-artery aneurysm in tocilizumabtreated children with Kawasaki's disease: To the editor. N Engl J Med. https://doi.org/10.1056/NEJMc1709609

\section{Publisher's Note}

Springer Nature remains neutral with regard to jurisdictional claims in published maps and institutional affiliations. 